\title{
The Hippo Signaling Pathway Regulates Ovarian Function via the Proliferation of Ovarian Germline Stem Cells
}

\author{
Haifeng Yea,b Xiaoyan Li $i^{a, b} \quad$ Tuochen Zheng ${ }^{c}$ Chuan Hua ${ }^{a, b}$ Zezheng Pan ${ }^{a, b}$ \\ Jian Huang ${ }^{a, b}$ Wei Lic Jia Lia,b Yuehui Zheng ${ }^{a, b}$

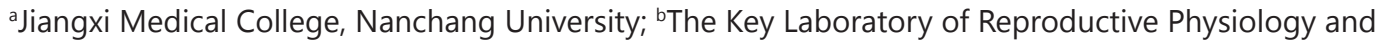 \\ Pathology of Jiangxi Provincial; 'School of the 1st Clinical Medical Sciences, Jiangxi Medical College, \\ Nanchang University, Nanchang, Jiangxi, PR China
}

\section{Key Words}

Hippo signaling pathway • YAP1 • Ovarian germline stem cells • Ovarian function • Mice

\begin{abstract}
Objective: To improve the separation, identification and cultivation of ovarian germline stem cells (OGSCs), to clarify the relationship between the Hippo signaling pathway effector YAP1 and the proliferation and differentiation of OGSCs in vitro and to identify the major contribution of Hippo signaling to ovarian function. Methods: Two-step enzymatic separation processes and magnetic separation were used to isolate and identify OGSCs by determining the expression of Mvh, Oct4, Nanog, Fragilis and Stella markers. Then, YAP1, as the main effector molecule in the Hippo signaling pathway, was chosen as the target gene of the study. Lentivirus containing overexpressed YAP1 or a YAP1-targeted shRNA was transduced into OGSCs. The effects of modulating the Hippo signaling pathway on the proliferation, differentiation, reproduction and endocrine function of ovaries were observed by microinjecting the lentiviral vectors with overexpressed YAP1 or YAP1 shRNA into infertile mouse models or natural mice of reproductive age. Results: (1) The specific expression of Mvh, Oct4, Nanog, Fragilis and Stella markers was observed in isolated stem cells. Thus, the isolated cells were preliminarily identified as OGSCs. (2) The co-expression of LATS2, MST1, YAP1 and MVH was observed in isolated OGSCs. Mvh and Oct4 expression levels were significantly increased in OGSCs overexpressing YAP1 compared to GFP controls. Consistently, Mvh and Oct4 levels were significantly decreased in cells expressing YAP1-targeted shRNA. (3) After 14-75 days of YAP1 overexpression in infertile mouse models, we detected follicle regeneration in ovaries, the activation of primordial follicles and increased birth rate, accompanied by increasing levels of E2 and FSH. (4) However, we detected decreasing follicles in ovaries, lower birth rate, and decreasing E2 and FSH in serum from healthy mice of reproductive age following YAP1 shRNA expression. Conclusion: Methods for the isolation, identification and culture of OGSCs were successfully established. Further results indicate that isolated OGSCs can specifically recognize Hippo signaling molecules and that manipulation of YAP1 expression can be used to regulate the proliferation and differentiation of OGSCs, as well as ovarian function in mice. This study suggests that the Hippo signaling pathway may represent a new molecular target for the regulation of mouse ovarian functional remodeling.




\section{Cellular Physiology Cell Physiol Biochem 2017;41:1051-1062 \\ and Biochemistry \begin{tabular}{c|c} 
DOI: 10.1159/000464113 \\
Published onnine: February 27, 2017 www.karger.com/cpb
\end{tabular}}

Ye et al.: The YAP1 Regulates Ovarian Function via the Proliferation of $\mathrm{OGSC}_{\mathrm{s}}$

\section{Introduction}

Contemporary studies have challenged the traditional view that mammalian females produce a finite number of oocytes in the prenatal phase of life and that this production ceases after birth $[1,2]$. In contrast, studies have successfully isolated and cultured ovarian germline stem cells (OGSCs), which produce oocytes and primordial follicles throughout an individual's lifetime [3-6]. In addition, several research groups have confirmed the presence of stem cells in the ovarian surface epithelium (OSE) of patients with infertility who have no mature follicles and oocytes [7-9].

In mammals, ovarian aging is a continuous process from birth to death, and in humans, this process can have long-term effects not only on reproductive capabilities but also on general health, with the potential to lead to psychological distress and depression, decreased sexual and general well-being, autoimmune disorders, osteoporosis, ischemic heart disease and increased risk for mortality [10]. The known causes of pathological ovarian aging include genetic aberrations, autoimmune ovarian damage and environmental influences $[10,11]$. Meanwhile, the cause of physiological ovarian aging is simply chronological aging [12]. Ovarian aging in general is one of the most important factors affecting female fertility and is accompanied by the typical clinical symptoms of increased gonadotropin levels and amenorrhea with hypoestrogenism $[10,13,14]$.

It has been shown that germline stem cells (GSCs) exist in both juvenile and adult mouse ovaries, and they are thought to be useful for recovery of ovarian function and even to reverse ovarian aging [15]. An increasing number of studies indicate that the Hippo signaling pathway is crucial for the self-renewal and differentiation of stem cells [16-20]. Furthermore, the Hippo signaling pathway plays an important role in the regulation of follicular growth [21]. In addition, the OSE layer has cell-cell contacts and local signaling that may be associated with GSC growth and development [22]. Importantly, evidence suggests that the Hippo signaling pathway is spatiotemporally correlated with the size of the primordial follicle pool [23] and that the existence of OGSCs in the mouse OSE and the expression of OGSCs markers are correlated with both the age of the mouse and with Hippo signaling molecules [24].

To our knowledge, there is no information on the effects of the Hippo pathway on the proliferation and differentiation of OGSCs and ovarian functional remodeling during aging in mouse models. In the current study, we isolated OGSCs via a two-step enzymatic separation method and magnetic-activated cell sorting (MACS), after which OGSCs were incubated with improved methods. The expression of YAP1, the main effector molecule in the Hippo signaling pathway, was manipulated in these cells via lentiviral overexpression or shRNA knockdown in vitro. In addition, YAP1 expression was modulated by microinjection of these constructs into the ovaries of infertile mouse models and healthy mice of reproductive age, to observe how YAP1 influences the proliferation and differentiation of OGSCs as well as ovarian reproductive and endocrine function in vivo. We first separated OGSCs by the two-step enzyme separation method and magnetic activated cell sorting (MACS) and then cultivated the OGSCs with improved methods. We chose the main effector molecule YAP1 in the Hippo signaling pathway as the target gene in the study and then observed what YAP1 can do to the proliferation, differentiation, ovarian reproductive function and endocrine function of the ovaries by micro-injecting over-expressed effector molecule YAP1 vectors and relevant shRNA interfering lentivirus vectors to transfect the infertile models and the healthy mice of reproductive age, respectively. Both over-expressed YAP1 and relevant shRNA interfering lentivirus vectors are transfected to OSCSs in vitro first.

\section{Materials and Methods}

Samples

60 two-month-old (2M) Kun Ming (KM) female mice that weighed approximately $30 \mathrm{~g}$ and 3-day-old (3d) KM female mice were obtained from the Centre of Experimental Animals, Nan Chang University (Nan 


\section{Cellular Physiology Cell Physiol Biochem 2017;41:1051-1062 and Biochemistry Published \begin{tabular}{l|l} 
DOI: 10.1159/000464113 2017 The Author(s). Published by S. Karger AG, Basel \\
www.karger.com/cpb
\end{tabular}

Chang, China). All the mice were fed and cared for according to the National Research Council Guide for Care and Use of Laboratory Animals. In addition, all the relevant animal experimental operations are under the guidance of the Institutional Animal Care and Use Committee. All animal feeding and nursing were in accordance with the National Research Council Guide for Care and Use of Laboratory Animals. All the animal experiment operations were conducted under the guidance of the IACUC. The $302 \mathrm{M}$ KM female mice were treated with an intraperitoneal injection of cyclophosphamide $(120 \mathrm{mg} / \mathrm{kg})$ and busulfan $(12 \mathrm{mg} / \mathrm{kg})$ to induce pathological ovarian aging, 15 used as the lentiviral YAP1 overexpressing (hYAP) group and the rest as the control-hGFP group. The 15 natural 2M KM female mice were used as the lentiviral YAP1 shRNA (shYAP) and the another 15 as the groupcontrol-shGFP, and between 8 and 10 3-day-old (3d) KM female mice were used for the isolation of OGSCs.

\section{Isolation and culture of OGSCS}

Between 16 and 20 ovaries were collected from 3-day-old KM female mice, washed with ice-cold PBS, and then minced into small pieces. Two-step enzymatic tissue dissociation and cell culture were performed as described previously [5]. The feeder for OGSCs culturing is SIM-6-thiogunanie-oualiain (STO) cell line (ATCC, Manassas, VA, USA).

\section{Cell proliferation assay}

Cell proliferation was determined using the Cell Counting Kit-8 (CCK-8; Dojindo, Tokyo, Japan). A total of $3 \times 10^{3}$ cells/well were seeded into 96-well plates and then treated with hYAP and shYAP. In all, $10 \mu \mathrm{L}$ of CCK-8 solution was added to each well and incubated for 1 hour. The absorbance of each well was measured at $450 \mathrm{~nm}$ using a microplate reader. The groups were as follows: lentiviral YAP1 overexpression group (hYAP): $1.02 \times 10^{9}$ (transduction unit, TU) with YAP1 virions; lentiviral YAP1 shRNA group (shYAP): $8 \times 10^{8} \mathrm{TU}$ with YAP1 virions; lentiviral empty control vector group (sh-control/control): $7.89 \times 10^{9}$ TU without YAP1 virions.

\section{Real-time PCR and quantitative real-time PCR}

Total RNA was extracted from the OGSCs using Tri-reagent (TaKaRa, Japan). The whole protocol was performed according to previously described methods [24]. Total RNA (1 $\mu$ g in a $25-\mu \mathrm{L}$ reaction) was reversetranscribed using the PrimeScriptTM RT reagent Kit with gDNA Eraser (TaKaRa, RR047A). For RT-PCR, 35 PCR cycles were performed using Permix Taq (Takara Taq version 2.0 plus dye, RR901) with primer sets specific for each gene (Table 1). The housekeeping gene we used is glyceraldehyde-3-phosphate dehydrogenase (GAPDH). Samples were resolved through $2 \%$ agarose gels and run under the same experimental conditions. DNA bands were detected using ethidium bromide (EB) staining. For quantitative real-time PCR, amplification was performed on an ABI7000 PCR instrument (Applied Biosystems, Foster City, CA, USA)

using three-stage program parameters provided by the manufacturer, as follows: $2 \mathrm{~min}$ at $50^{\circ} \mathrm{C}, 2 \mathrm{~min}$ at $95^{\circ} \mathrm{C}$, and then 40 cycles of $15 \mathrm{~s}$ at $95^{\circ} \mathrm{C}$ and $60 \mathrm{~s}$ at $60^{\circ} \mathrm{C}$, followed by a final cooling step at $4^{\circ} \mathrm{C}$. The primers designed for amplifying are listed in Table 1 . The reaction was carried out in a $20-\mu \mathrm{l}$ volume containing $10 \mu \mathrm{L}$ of SYBR green Master Mix (Life Technologies, USA), $0.5 \mu \mathrm{L}$ of cDNA, $0.6 \mu \mathrm{L}$ of $50 \mathrm{mM} \mathrm{MgCl}$, $1.6 \mu \mathrm{L}$ of $10 \mu \mathrm{M}$ primers, and sterilized distilled water up to $20 \mu \mathrm{L}$. All assays were independently repeated at least in triplicate, and GAPDH was used as the reference gene.
Table 1. The sequences of primers used for RT-PCR

\begin{tabular}{|c|c|}
\hline Gene & Primer sequence \\
\hline \multicolumn{2}{|c|}{ GAPDH 5'-CAGGTGGTCTCCTCTGACTT-3' (forward) } \\
\hline & 5'-CCAAATTCGTTGTCATACCA-3'(reverse) \\
\hline \multirow[t]{2}{*}{ MVH } & 5'- GTGTATTATTGTAGCACCAACTCG-3' (forward) \\
\hline & 5'-CACССТTGTACTATCTGTCGAACT-3'(reverse) \\
\hline \multirow[t]{2}{*}{ OCT4 } & 5'- AGCTGCTGAAGCAGAAGAGG-3' (forward) \\
\hline & 5’- GGTTCTCATTGTTGTCGGCT-3' (reverse) \\
\hline \multirow[t]{2}{*}{ Fragilis } & 5'- CTGGTCCCTGTTCAATACACTCTT-3' (forward) \\
\hline & 5'- CAGTCACATCACCCACCATCTT-3' (reverse) \\
\hline \multirow[t]{2}{*}{ Stella } & $5^{\prime}$ - CCCAATGAAGGACCCTGAAAC-3' (forward) \\
\hline & 5'- AATGGCTCACTGTCCCGTTCA-3' (reverse) \\
\hline \multirow[t]{2}{*}{ Nanog } & $5^{\prime}$ - TCTCCTCGCCCTTCCTCTGA -3' (forward) \\
\hline & 5'- TCCGCATCTTCTGCTTCCTG -3' (reverse) \\
\hline \multirow[t]{2}{*}{ YAP1 } & 5'-TCCAACCAGCAGCAGCAAAT-3' (forward) \\
\hline & 5'-TTCCGTATTGCCTGCCTGCCGAAA-3' (reverse) \\
\hline
\end{tabular}




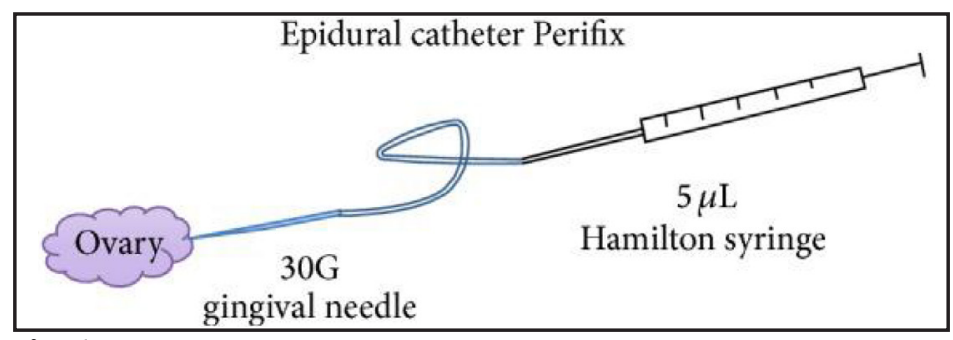

Fig. 1. The picture of microinjection in vitro [25].

Alkaline phosphatase and immunofluorescence staining

Alkaline phosphatase (AP) staining was performed using an Alkaline Phosphatase Detection kit (Sigma) according to the manufacturer's instructions.

For immunofluorescence, the procedure was performed according to previously published protocols [24]. The primary antibodies used in this study were anti-LATS2 (1:100, ab54073), anti-MST1 (1:100, ab51134), anti-YAP1 (1:100, ab56701), anti-S127 phosphorylated-YAP1 (1:100, ab76252), anti-OCT4 (1:100, ab18976) and anti-MVH (1:100, ab27591). The secondary antibodies used in this study were goat anti-mouse and goat anti-rabbit IgG conjugated with fluorescein isothiocyanate at a 1:200 dilution (Proteintech, China). Finally, the tissues were stained with DAPI. The samples were imaged with a NIKON Eclipse 80i microscope.

\section{Microinjection of lentivirus in vitro}

All the test mice were treated without food but with freely available water for 12 hours prior to the experiment. Mice were anesthetized with $1 \%$ sodium pentobarbital via intraperitoneal injection and fixed them in a prone position. A $1.0 \mathrm{~cm}$ longitudinal incision was cut at the side of the spine through the subcutaneous tissue, muscular layer and peritoneum, through which both ovaries were removed. Different doses of lentiviral particles were injected into the ovarian envelope under the microscope in vitro; in the control group, lentivirus was replaced with the same volume of $0.9 \%$ normal saline solution. (Fig. 1) [25]. After injections, the ovaries were placed back into the abdomen and incisions were disinfected and sutured.

\section{Serum hormone level measurements}

Fifteen days after infection, blood was drawn from the orbital socket and serum was collected. The levels of E2 and FSH were measured by the biological company.

Data presentation and statistical analysis

GraphPad Prism software (GraphPad Software, La Jolla, CA) was used for statistical analysis. The statistical significance of differences among groups was determined using Student's t test. Probability values of $\mathrm{p}<0.05$ were considered significant; $\mathrm{p}<0.01$ and $\mathrm{p}<0.001$ were both considered extremely significant.

\section{Results}

\section{The isolation and identification of OGSCs}

We isolated OGSCs from the ovaries of 3-day-old KM mice using a two-step enzymatic method and magnetic beads with MVH-specific antibodies. This resulted in the isolation of separated oval reproductive cells, approximately 15-20 $\mu \mathrm{m}$ in diameter (Fig. 2A). The cells were not numerous when initially isolated, and their proliferation cycle was approximately 5-7 days. When the cells reached 3-4 generations, they began to multiply more robustly, distributing like colonies.

After 15 days in culture, cellular mRNA was extracted and analyzed by PCR, which showed positive expression of the known OGSCs markers Mvh, Oct4, Nanog, Fragilis and Stella (Fig. 2B). In addition, we immunostained for the reproductive cell marker MVH and specific stem cell marker OCT4 and both were expressed positively in the separated cells. These reproductive cells also had the potential to replicate, as verified via EDU and AP staining (Fig. 2C,D,E). Thus, these cells can be preliminarily identified as OGSCs. 
A
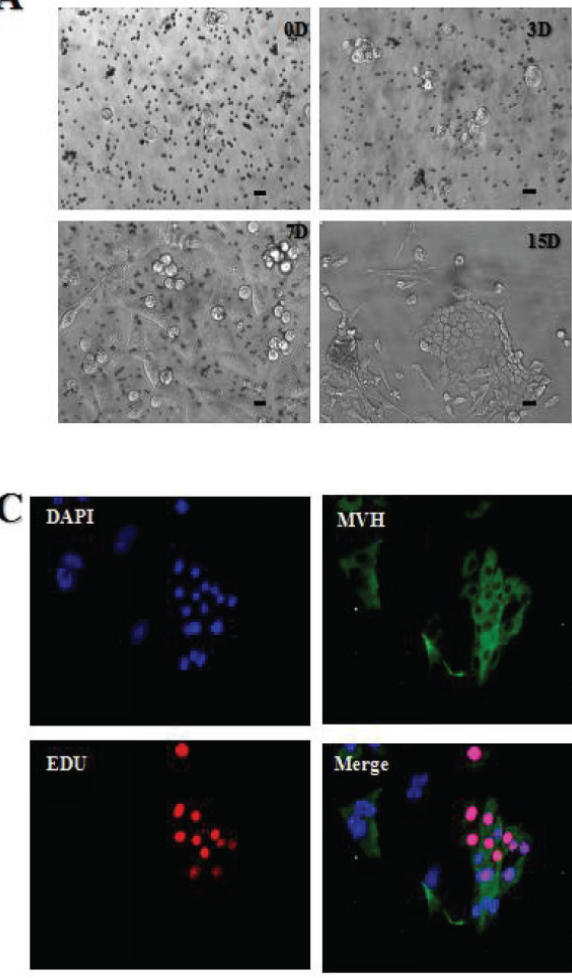
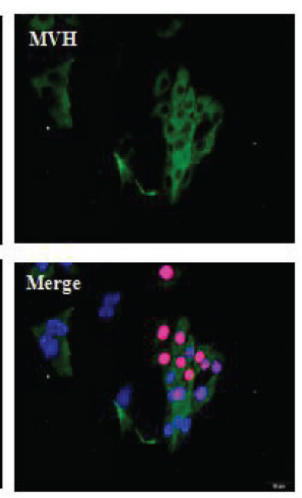

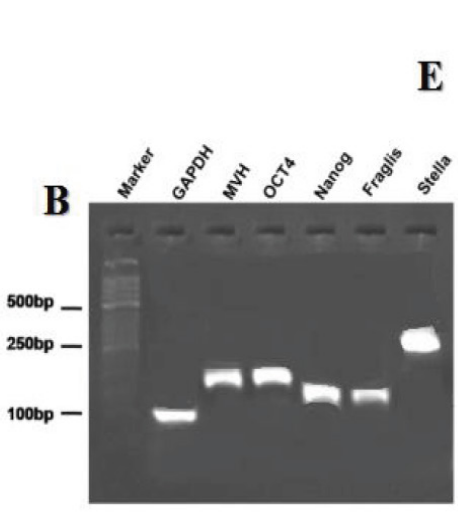

$\mathbf{E}$
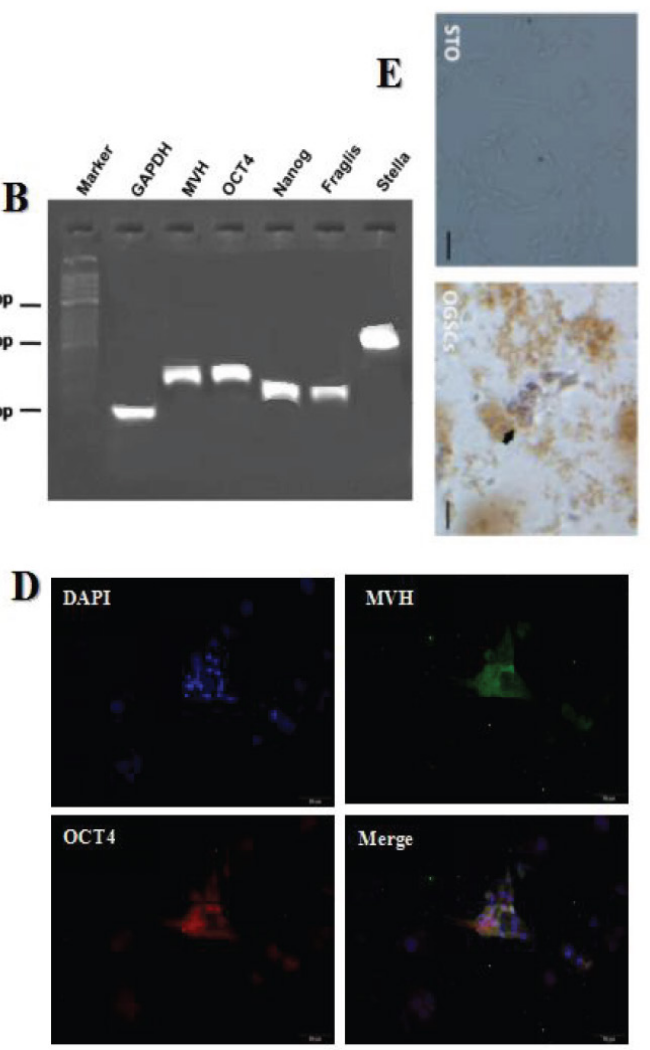

Fig. 2. A: The different morphological characteristics of isolated germ cells after 0 days, 3 days, 7 days and 15 days. Scale bar: $20 \mu \mathrm{m}$. B: RT-PCR analysis of germ cell markers (M: 100 bp lane marker). Immunofluorescence of germ cells cultured in vitro for 15 days with MVH and OCT4. C: EDU, MVH and DAPI. Scale bars: 10 $\mu \mathrm{m}$. D: OCT4, MVH and DAPI. Scale bars: $20 \mu \mathrm{m}$. E: The alkaline phosphatase (AP) staining of isolated germ cells. Scale bars: $20 \mu \mathrm{m}$.

Hippo signaling molecules are expressed in OGSCs

Using immunostaining, we discovered that three key molecules in the Hippo signaling pathway (LATS2, MST1 and YAP1) are all expressed in OGSCs. In addition, LATS2, MST1 and YAP1 all co-express with MVH in OGSCs (Fig. 3).

Overexpression of YAP1 affects the proliferation and differentiation of OGSCS

We transduced 3rd-4th generation OGSCs in vitro with YAP1-overexpressing lentivirus and observed the effects after 48 hours of expression. All transfected cells showed obvious green immunofluorescence expression, indicating high GFP expression and confirming successful lentivirus infection (Fig. 4A). 4 days after viral infection, cells were passage cultivated with lentivirus in 96-well plates. After cell adherence, the cells' multiplication efficiency was measured with a CCK-8 Cell Proliferation Kit. The results indicated that cells overexpressing YAP1 had a higher absorbance value compared to the blank groups ( $\mathrm{p}<0.001$; Fig. 4B), suggesting that YAP1 promotes the proliferation of OGSCs in vitro.

Next, we extracted total mRNA from the cultured cells 72 hours after transduction and quantified the expression of Hippo pathway and OGSCs marker genes via fluorescent real time PCR (RT-PCR). As expected, the effector molecule Yap1 was more highly expressed in the lentivirus-mediated YAP1 overexpression group comparing to the control group. In addition, $M v h$ and Oct4, specific markers of OGSCs, were also more highly expressed in the YAP1 overexpression group $(\mathrm{p}<0.05, \mathrm{p}<0.01$ ) (Fig. $4 \mathrm{C}$ ), suggesting YAP1 may promote the differentiation of the OGSCs cell fate.

\section{KARGER}


Fig. 3. Immunofluorescence of OGSCs markers and Hippo signaling components. Scale bars: $20 \mu \mathrm{m}$.

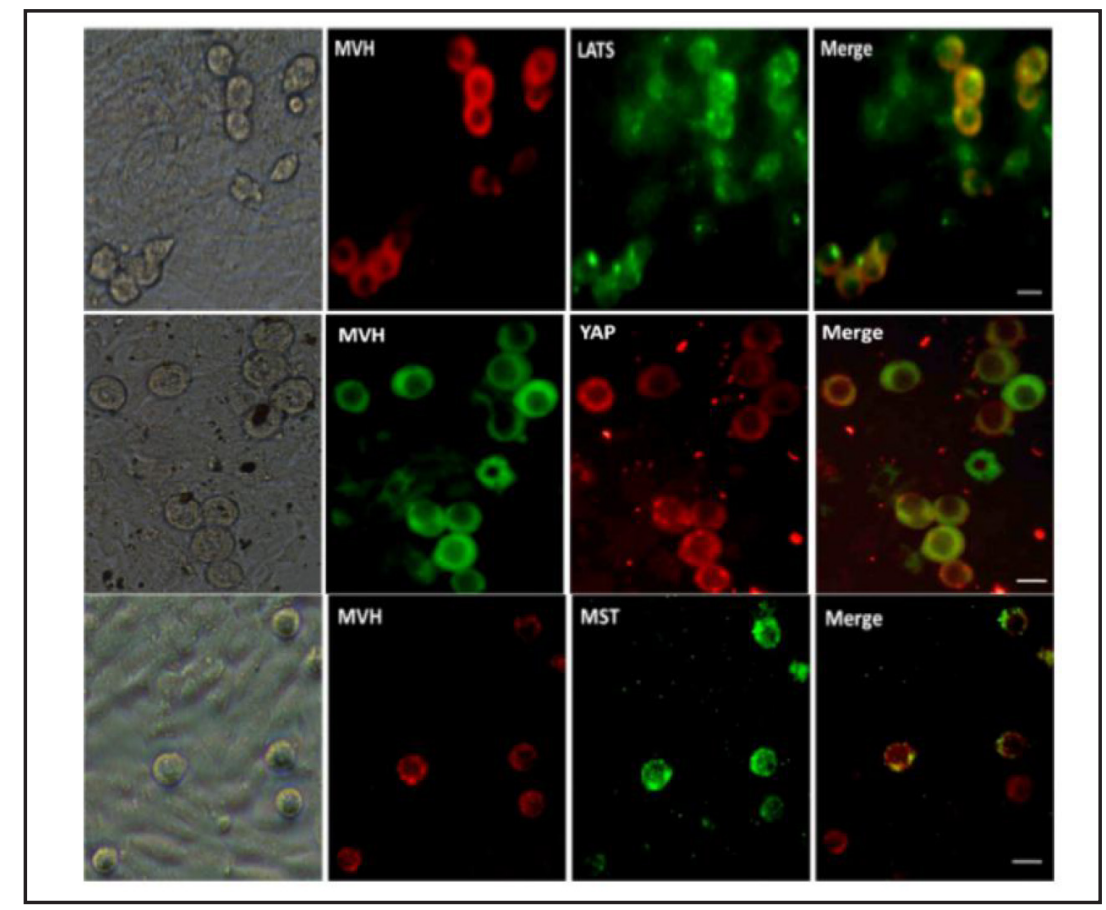

Fig. 4. The development of OGSCs after YAP1 overexpression in vitro A: The expression of GFP in different treatment groups. B: The change in cell proliferation as detected by CCK- 8 assay. The results are presented as the mean \pm SD. $* \mathrm{p}$ $<0.05, * * \mathrm{p}<0.01, * * * \mathrm{p}$ C $<0.001$, compared with the control group. Scale bars: $20 \mu \mathrm{m}$. C: The data analyzed by RT-qPCR. The results are presented as the mean \pm SD. $* \mathrm{p}$ $<0.05,{ }^{* *} \mathrm{p}<0.01,{ }^{* * *} \mathrm{p}$ $<0.001$, compared with the control group.

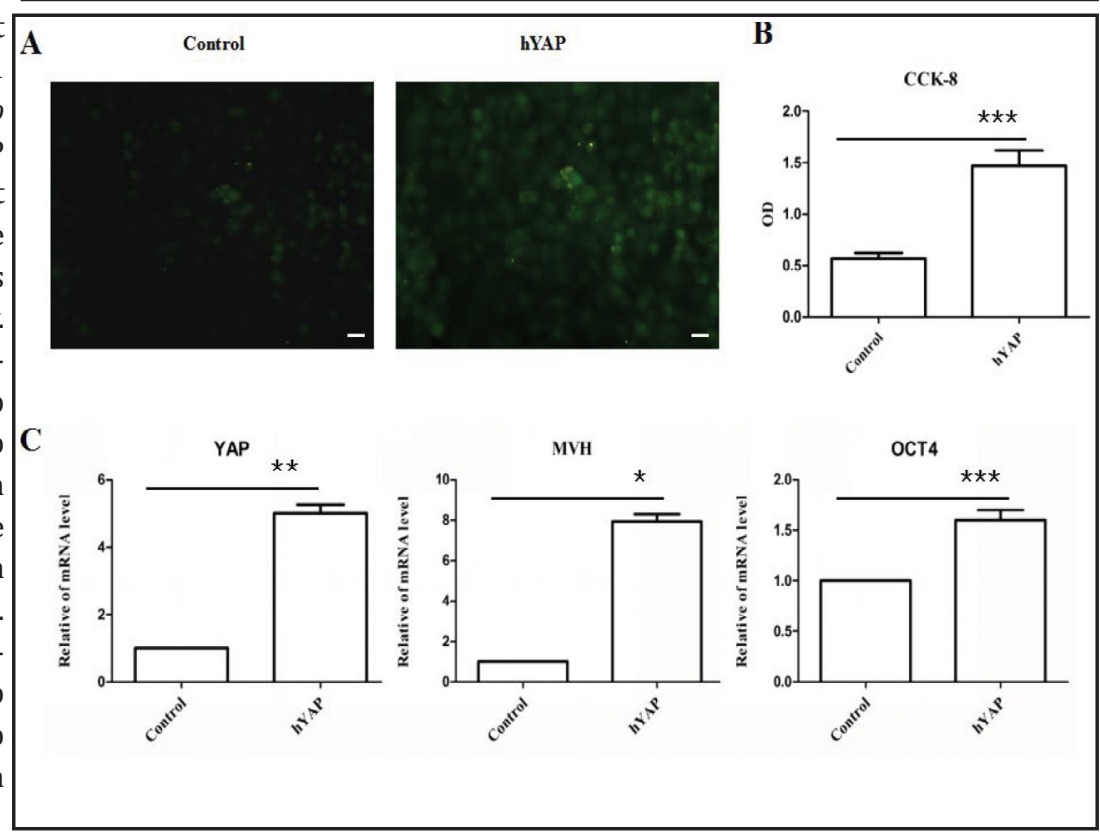

The effect of YAP1 knockdown on the proliferation and differentiation of OGSCs

To confirm the role of YAP1 in regulating OGSCs proliferation and differentiation, we also transduced cells with a lentivirus expressing a YAP1 shRNA to decrease its expression. In these cells, we observed clear GFP expression 48 hours following transduction, confirming successful viral infection (Fig. 5A). Consistent with our observations with YAP1 overexpression, the multiplication efficiency of YAP1 knockdown cells was dramatically lower than that of control cells ( $p<0.001$;) (Fig. 5B). This demonstrates that YAP1 is necessary for the proliferation of OGSCs in vitro.

By immunostaining, we confirmed that Yap1 expression was lower YAP1 shRNA cells compared to control cells (Fig. 5C). In addition, the specific OGSCs markers Mvh and Oct4 were significantly less expressed in the YAP1 shRNA cells, further supporting a role for YAP1 in differentiation $(\mathrm{p}<0.05, \mathrm{p}<0.01, \mathrm{p}<0.001)$. 


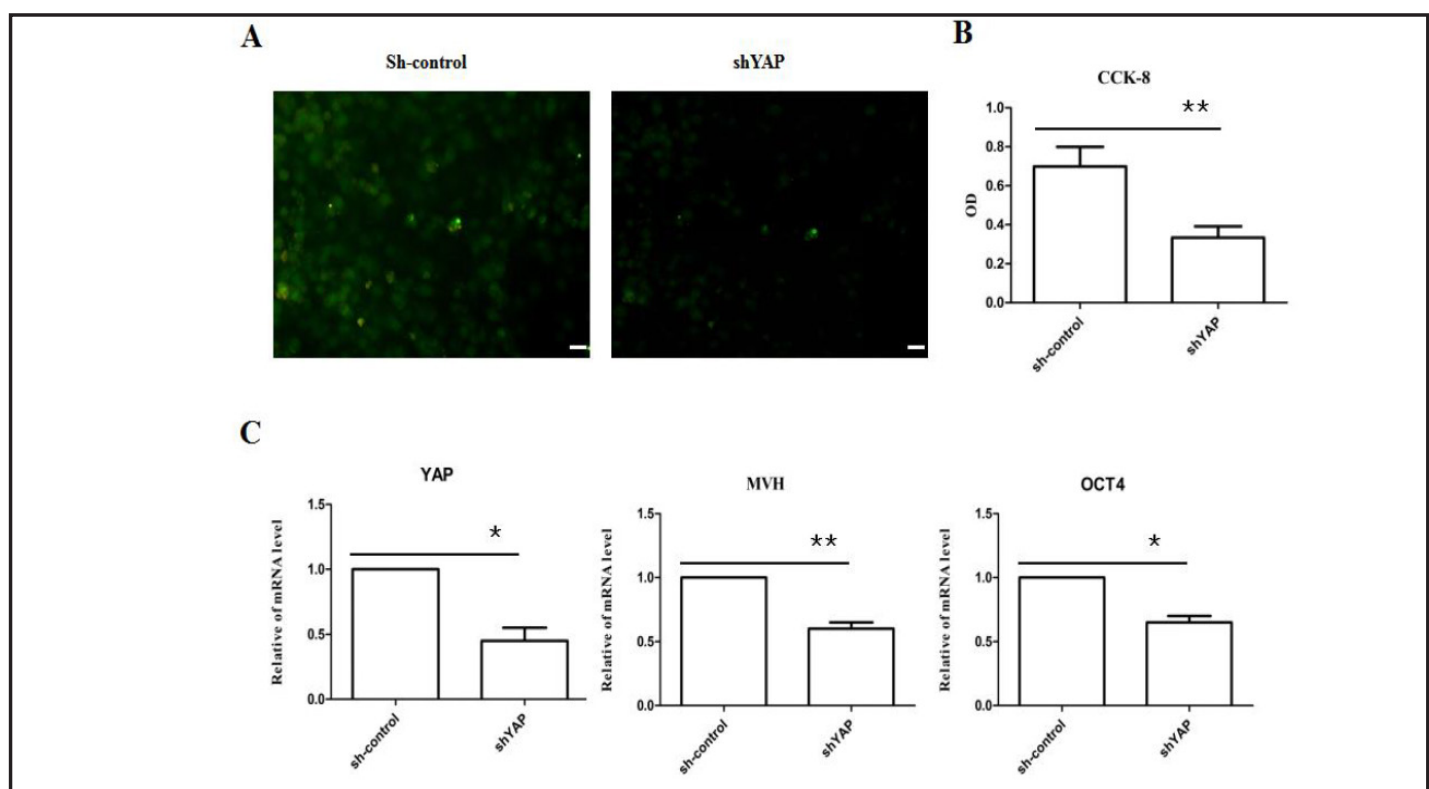

Fig. 5. The development of OGSCs after YAP1 knockdown in vitro. A: The expression of GFP in different treated groups. B: The changes in cell proliferation as detected by CCK-8 assay. The results are presented as the mean \pm SD. ${ }^{*} \mathrm{p}<0.05,{ }^{* *} \mathrm{p}<0.01,{ }^{* * *} \mathrm{p}<0.001$, compared with the control group. Scale bars: $100 \mu \mathrm{m}$. C: The data analyzed by RT-qPCR. The results are presented as the mean \pm SD. ${ }^{*} p<0.05,{ }^{* *} \mathrm{p}<0.01,{ }^{* * *} \mathrm{p}<0.001$, compared with the control group.

A

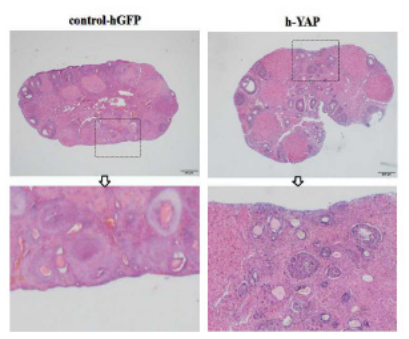

C

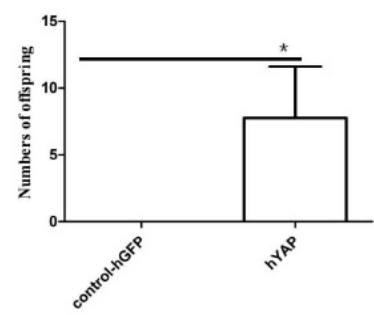

B
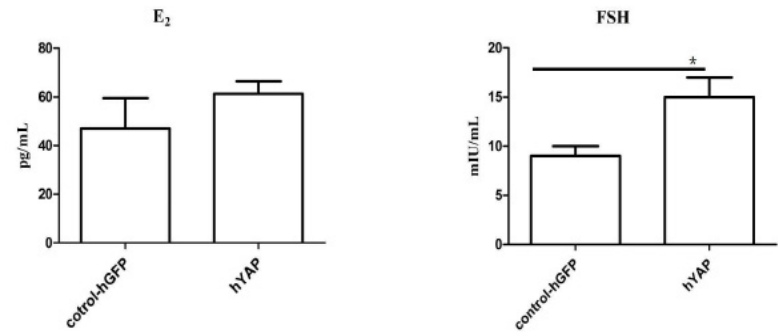

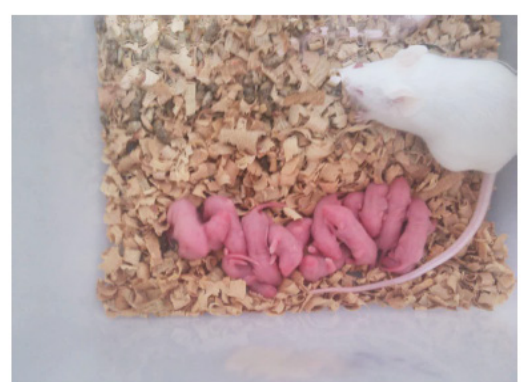

Fig. 6. Ovarian function after YAP1 overexpression in vitro. A: Photomicrograph H\&E stained from histological ovary sections: experimental groups euthanized on day 14 (Upper: 10×; Lower: 40×). B: The expression changes of E2 and FSH after ovarian YAP1 overexpression. The results are presented as the mean \pm SD. ${ }^{*} \mathrm{p}<$ 0.05 , compared with the GFP group. C: The numbers of offspring produced by post-transplantation mouse recipients overexpressing YAP1 in ovaries. The results are presented as the mean $\pm S D . * p<0.05$, compared with the GFP group. 


\begin{tabular}{ccc}
\hline Groups & Injection of days Numbers of offspring \\
\hline hYAP & $30 \mathrm{~d}$ & 10 \\
hYAP & $40 \mathrm{~d}$ & 5 \\
hYAP & $60 \mathrm{~d}$ & 4 \\
hYAP & $75 \mathrm{~d}$ & 12 \\
control-hGFP & $30 \mathrm{~d}$ & 0 \\
control-hGFP & $45 \mathrm{~d}$ & 0 \\
control-hGFP & $60 \mathrm{~d}$ & 0 \\
\hline
\end{tabular}

Table 2. The birth times and the number of offspring produced by post-transplantation-YAP mouse recipients by natural mating with a wild-type KM male

\begin{tabular}{ccc}
\hline Groups & \multicolumn{2}{c}{ Injection of days Numbers of offspring } \\
\hline shYAP & $30 \mathrm{~d}$ & 5 \\
shYAP & $45 \mathrm{~d}$ & 8 \\
shYAP & $60 \mathrm{~d}$ & 7 \\
shYAP & $75 \mathrm{~d}$ & 13 \\
control-shGFP & $30 \mathrm{~d}$ & $12(\times 2)$ \\
control-shGFP & $40 \mathrm{~d}$ & $15(\times 2)$ \\
control-shGFP & $45 \mathrm{~d}$ & $15(\times 4)$ \\
control-shGFP & $60 \mathrm{~d}$ & $16(\times 2)$ \\
\hline
\end{tabular}

Table 3. The birth times and the number of offspring produced by post-transplantation-shYAP mouse recipients by natural mating with a wild-type KM male
A
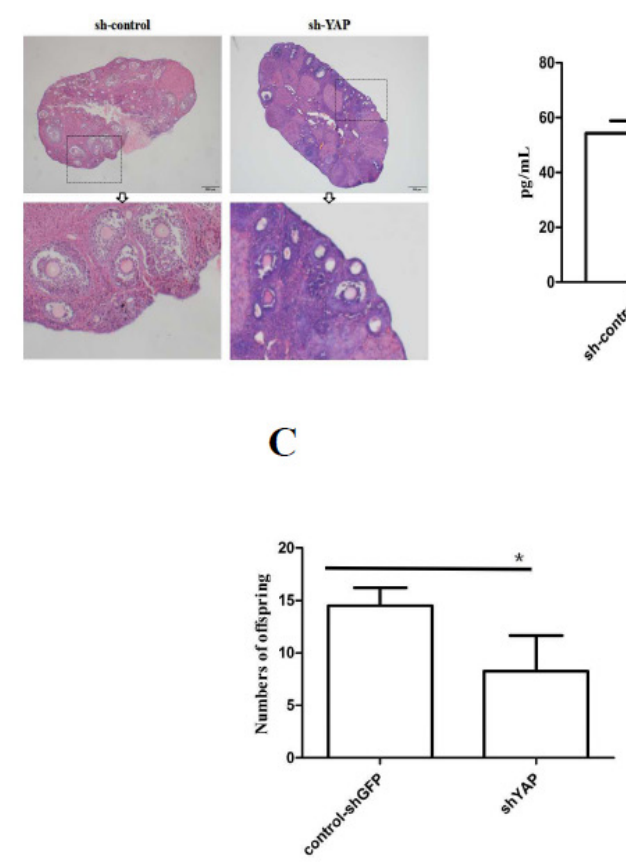

\section{B}
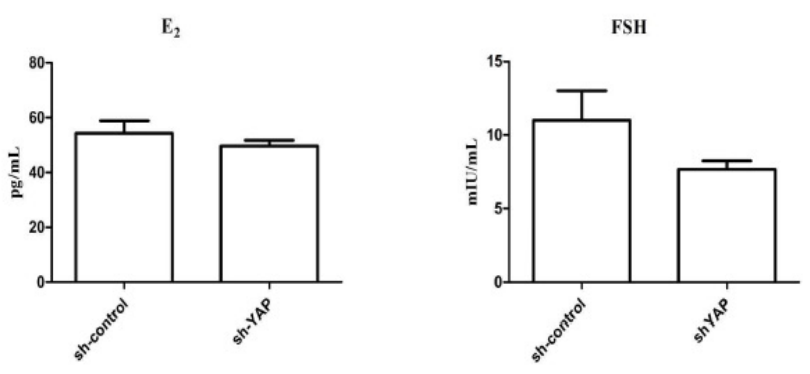

Fig. 7. Ovarian function after YAP1 knockdown in vitro. A: Photomicrograph H\&E stained from histological ovary sections: experimental groups euthanized on day 14 (Upper: 10x; Lower: 40x). B: The expression changes of E2 and FSH after ovarian YAP1 knockdown. The results are presented as the mean \pm SD. ${ }^{*} \mathrm{p}<$ 0.05, compared with the GFP group. C: The numbers of offspring produced by post-transplantation mouse recipients with ovarian YAP1 knockdown. The results are presented as the mean \pm SD. ${ }^{*} \mathrm{p}<0.05$, compared with the GFP group.

YAP1 regulates reproductive and endocrine function in ovarian tissue

We next microinjected the YAP1 overexpression or knockdown lentiviruses into the ovaries of female mice belonging to infertile mouse model, to confirm a role for YAP1 in the regulation of ovarian function. With YAP1 overexpression, the formation of primordial follicles was clearly visible, and the. thickness of the OSE increased (Fig. 6A). However, there was neither formation of primordial follicles nor any change in the thickness of the OSE in the control group.

After 15 days of lentivirus expression, serum was collected from the infected mice to measure the dynamic levels of E2 and FSH. The levels of E2 and FSH in serum of YAP1 
Ye et al.: The YAP1 Regulates Ovarian Function via the Proliferation of $\mathrm{OGSC}_{\mathrm{s}}$

overexpressing mice are both elevated compared to the control group, particularly the level of FSH $(\mathrm{p}<0.05)$ (Fig. 6B). These results suggest that high expression of YAP1 can induce the growth of oocytes and the proliferation of follicle cells.

We also assessed the mating behavior of formerly infertile female mice overexpressing ovarian YAP1. 15 days following ovarian microinjection, 2-3 female mice were placed in each cage, 12 mice survived in the YAP1 overexpressing group while 15 survived in the control group. 1-2 male mice were placed in each cage, and the mating, births, and number of offspring were observed for each group. The results are shown in Table 2. In the YAP1 overexpressing group, 4 female mice gave birth to 31 offspring at the 30th, 40th, 60th and 75th day, respectively, a birth rate of approximately 33\%. However, the control group did not produce any offspring, suggesting they remained infertile in the absence of YAP1 overexpression (Fig. 6C).

\section{The effects of YAP1 knockdown on ovarian and endocrine function}

We also microinjected lentivirus expressing the YAP1 shRNA into the ovaries of female wild-type mice and performed similar experiments to those described in Fig. 6. The number of follicles decreased in the ovaries of mice expressing YAP1 shRNA compared to the control group, but there was no change in the thickness of the OSE (Fig. 7A).

In addition, the expression levels of E2 and FSH in serum of the YAP1 ovarian knockdown mice declined $(\mathrm{p}<0.05)$ (Fig. 7B). These results demonstrate that decreased expression of YAP1 in ovaries can inhibit the growth of oocytes and the proliferation of follicle cells.

The results of mating experiments with the YAP1 ovarian knockdown mice are shown in Table 3. In total, 4 YAP1 ovarian knockdown mice gave birth to 33 offspring at the 30 th, 45 th, 60 th and 80th day, respectively, a birth rate of approximately $26.7 \%$. However, 10 mice in the control group gave birth to 135 offspring, demonstrating that decreased ovarian expression of YAP1 negatively impacts mating and reproduction (Fig. 7C).

\section{Discussion}

In 2004, Johnson et al. [2] found that female mammals can produce new oocytes constantly from birth until adulthood to renew the primordial follicle pool, a theory termed "neo-oogenesis." However, this discovery challenged the follicle-pool fixed theory that had dominated reproductive medicine for over 100 years. Since then, there has been an enhanced interest in OGSCs research. Although the existence of mammalian OGSCs remains controversial $[26,27]$, many groups have identified OGSCs in mammalian ovaries by stem cell culture in vitro, transgenesis and in vivo lineage tracing [28-30].

Previously, OGSCs have been considered difficult to isolate and cultivate in vitro, as there are many cell types in ovaries with a complicated growth and differentiation process. Compared to many ovarian cell types, there are relatively few OGSCs in ovaries and there are no specific surface markers for these cells [31,32]. Thus, the limiting factor for the study of OGSCs behavior and characteristics in vitro has been the separation and purification of these cells while maintaining their self-renewal and self-differentiation ability.

In this study, we overcame these limitations by first choosing specific antibody-tagged magnetic beads and the reproductive cell marker MVH to screen ovarian reproductive cells. We were then able to identify which cells in ovaries have stem cell characteristics and reproductive ability, combining $\mathrm{MVH}$, the reproductive-specific transcription factor OCT4, the specific reproductive cell marker EDU, and other factors. We found that OGSCs separated by this method are distributed like colonies in cell culture, similar to existing research reports $[5,33,34]$. With RT-PCR, we could detect the expression of cell markers at the 15th day in culture and found that these cells express Mvh, Fragilis and Stella mRNA, which indicates the separated cells are reproductive cells. The positive expression of Nanog and Oct4 indicates that these cells also have stem cell ability and pluripotency. In addition, these cells expressed positive for alkaline phosphatase when compared to STO cells used as a feeder layer. Thus,

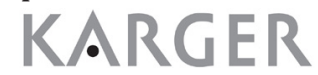




\section{Cellular Physiology Cell Physiol Biochem 2017;41:1051-1062

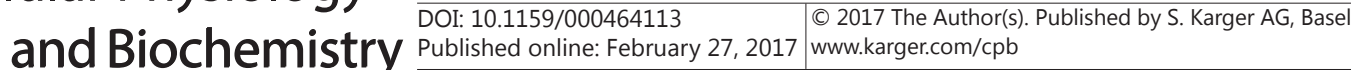

Ye et al.: The YAP1 Regulates Ovarian Function via the Proliferation of OGSC

we can conclude that the cells isolated by this method are primarily OGSCs, strong evidence for the existence of reproductive stem cells in mammalian ovaries after birth.

We chose immunofluorescence double labeling in this study to detect the co-expression of Hippo signaling proteins and MVH and identified that the Hippo signaling pathway is present in the separated reproductive cells. Lentiviruses stably insert target genes in the host genome, making this an ideal system for genetic engineering. Furthermore, lentiviral vectors have improved transduction efficiency, making it possible to stably express constructs longterm $[35,36]$. We used this system to infect OGSCs with YAP1 or shRNA against YAP1. After 48 hours, we observed the results by immunofluorescence, multiplication assay, and RT-PCR. Our results revealed that Yap1 overexpression increased the proliferation of OGSCs, as well as the expression of Yap1, Mvh and Oct4 mRNA. In contrast, YAP1 shRNA decreased cellular proliferation, as well as the expression of Yap1, Mvh and Oct4 mRNA. Thus, YAP1 regulates the proliferation and differentiation of OGSCs.

Recent evidence suggests that the Hippo-YAP pathway provides a new method for cancer treatment in clinical studies [37, 38]. However, the application of this signaling pathway to the treatment of ovarian diseases has not been tested. This research puts forward the idea that YAP1 can reconstruct the ovarian functions. Our results reveal that the expression levels of E2 and FSH increased obviously upon YAP1 overexpression via ovarian microinjection. In addition, some previously infertile mice gave birth to offspring at a birth rate of $33.3 \%$. This indicates that overexpression of YAP1 promote primordial follicle formation, inducing the growth of oocytes and the recovery of ovarian functions. Consistently, ovarian microinjection of YAP1 shRNA decreased the expression levels of E2 and FSH, and only 4 previously fertile mice gave birth, a rate of $26.7 \%$. In contrast, 10 fertile mice in the control group gave birth, which indicates that decreased expression of YAP1 can inhibit the proliferation and differentiation of OGSCs, maintain primordial follicles in quiescence, and influence the normal growth of oocytes and ovarian functions.

This study provides evidence that the Hippo effector molecule YAP1 regulates the proliferation and differentiation of OGSCs in vivo, clarifying the role of YAP1 in reconstructing physiological ovarian functions. This increases our understanding of the mechanisms of OGSCs development and may provide a more effective method for treating female infertility. Moreover, these results have great potential for the preservation and reproduction of animals, especially endangered species.

However, there are still some limitations in our research. First, we were not able to subculture the separated and purified OGSCs without limit via our in vitro culture system. In the meanwhile, stable over-expressed and low-expressed OGSCs can not be screened which also blocked our research. In addition, the great difficulty of cultivating and enriching the OGSCs in vitro, several factors during the micro-injecting operation procedure as well as the individual differences between the mice all have much impact on the research which can well-explain our limitations in our research. As we analyzed the causes, at first the culture conditions of cultivating OGSCs were extremely strict and complicated so that our experiments were much influenced by the cultivation environment. For example, both the trace growth factor and the external environment can influence the growth and the proliferation of OGSCs, making the cells hard to passage without limit. Secondly, the proliferation rate of OGSCs was quite slow and the amount of cells was small. So in the future experiment research, we will improve and make some adjustments on the cultivation system. In addition, we will choose to use the human ovaries as our experimental subjects to further prove the important role of Hippo signalling pathway in human OGSCs and ovarian functions remodeling.

\section{Acknowledgements}

The authors would like to thank Dr. Liping Zheng and Dr. Xuhui Zeng for technical assistance, We are thankful to the Life Sciences Institute of Nanchang University for providing a high standard of scientific research conditions, We also thank the members in our lab who participated in this study. 


\section{Cellular Physiology Cell Physiol Biochem 2017;41:1051-1062

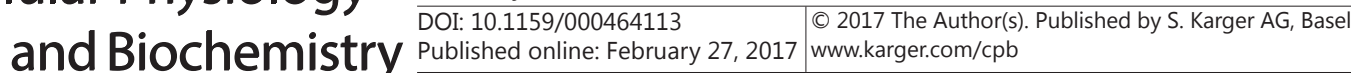

Ye et al.: The YAP1 Regulates Ovarian Function via the Proliferation of OGSC $\mathrm{s}$

This work was supported by the National Nature Science Foundation of China (No. $81360100,81260098,81671455$ ), the Natural Science Foundation of Jiangxi province (No. 20152ACB20003, 20142BAB205002, 20142BAB205069) and the Excellence 555 Engineering of Jiangxi province.

\section{Disclosure Statement}

The authors declare no conflicts of interest.

\section{References}

1 Evron A, Blumenfeld Z: Ovarian Stem Cells-the Pros and Cons. Clin Med Insights Reprod Health 2013;7:4347.

2 Johnson J, Canning J, Kaneko T, Pru JK, Tilly JL: Germline stem cells and follicular renewal in the postnatal mammalian ovary. Nature 2004;428:145-150.

3 Parvari S, Abbasi M, Abbasi N, Malek VG, Amidi F, Aval FS, Roudkenar MH, Izadyar F: Stem cell isolation by a morphology-based selection method in postnatal mouse ovary. Arch Med Sci 2015;11:670-678.

4 Woods DC, Tilly JL: Isolation, characterization and propagation of mitotically active germ cells from adult mouse and human ovaries. Nat Protoc 2013;8:966-988.

- Z Zou K, Yuan Z, Yang Z, Luo H, Sun K, Zhou L, Xiang J, Shi L, Yu Q, Zhang Y, Hou R, Wu J: Production of offspring from a germline stem cell line derived from neonatal ovaries. Nat Cell Biol 2009;11:631-636. Khosravi-Farsani S, Amidi F, Habibi Roudkenar M, Sobhani A: Isolation and enrichment of mouse female germ line stem cells. Cell J 2015;16:406-415.

7 Virant-Klun I: Postnatal oogenesis in humans: a review of recent findings. Stem Cells Cloning 2015;8:49-60. Virant-Klun I, Skutella T, Kubista M, Vogler A, Sinkovec J, Meden-Vrtovec H: Expression of pluripotency and oocyte-related genes in single putative stem cells from human adult ovarian surface epithelium cultured in vitro in the presence of follicular fluid. Biomed Res Int 2013;2013:861460.

-9 Virant-Klun I, Skutella T, Stimpfel M, Sinkovec J: Ovarian surface epithelium in patients with severe ovarian infertility: a potential source of cells expressing markers of pluripotent/multipotent stem cells. J Biomed Biotechnol 2011;2011:381928.

10 Goswami D, Conway GS: Premature ovarian failure. Horm Res 2007;68:196-202.

11 Ye H, Li X, Zheng T, Liang X, Li J, Huang J, Pan Z, Zheng Y: The effect of the immune system on ovarian function and features of ovarian germline stem cells. Springerplus 2016;5:990.

12 Sukur YE, Kivancli IB, Ozmen B: Ovarian aging and premature ovarian failure. J Turk Ger Gynecol Assoc 2014;15:190-196.

13 Broekmans FJ, Soules MR, Fauser BC: Ovarian aging: mechanisms and clinical consequences. Endocr Rev 2009;30:465-493.

14 Jacobsen BK, Heuch I, Kvale G: Age at natural menopause and all-cause mortality: a 37-year follow-up of 19,731 Norwegian women. Am J Epidemiol 2003;157:923-929.

15 Shi L, Wu J: Epigenetic regulation in mammalian preimplantation embryo development. Reprod Biol Endocrinol 2009;7:59.

16 Polesello C, Tapon N: Salvador-warts-hippo signaling promotes Drosophila posterior follicle cell maturation downstream of notch. Curr Biol 2007;17:1864-1870.

17 Chen HJ, Wang CM, Wang TW, Liaw GJ, Hsu TH, Lin TH, Yu JY: The Hippo pathway controls polar cell fate through Notch signaling during Drosophila oogenesis. Dev Biol 2011;357:370-379.

18 Varelas X, Miller BW, Sopko R, Song S, Gregorieff A, Fellouse FA, Sakuma R, Pawson T, Hunziker W, McNeill H, Wrana JL, Attisano L: The Hippo pathway regulates Wnt/beta-catenin signaling. Dev Cell 2010;18:579591.

19 Yan Y, Denef N, Tang C, Schupbach T: Drosophila PI4KIIIalpha is required in follicle cells for oocyte polarization and Hippo signaling. Development 2011;138:1697-1703.

20 Heallen T, Zhang M, Wang J, Bonilla-Claudio M, Klysik E, Johnson RL, Martin JF: Hippo pathway inhibits Wnt signaling to restrain cardiomyocyte proliferation and heart size. Science 2011;332:458-461. 


\section{Cellular Physiology Cell Physiol Biochem 2017;41:1051-1062

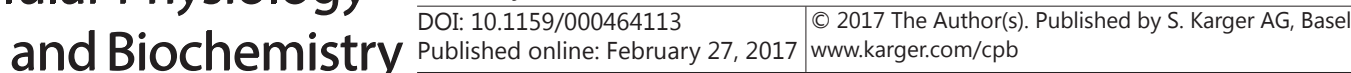

21 Kawamura K, Cheng Y, Suzuki N, Deguchi M, Sato Y, Takae S, Ho CH, Kawamura N, Tamura M, Hashimoto S, Sugishita Y, Morimoto Y, Hosoi Y, Yoshioka N, Ishizuka B, Hsueh AJ: Hippo signaling disruption and Akt stimulation of ovarian follicles for infertility treatment. Proc Natl Acad Sci U S A 2013;110:17474-17479. Kezele P, Nilsson E, Skinner MK: Cell-cell interactions in primordial follicle assembly and development. Front Biosci 2002;7:d1990-1996.

23 Xiang C, Li J, Hu L, Huang J, Luo T, Zhong Z, Zheng Y, Zheng L: Hippo signaling pathway reveals a spatiotemporal correlation with the size of primordial follicle pool in mice. Cell Physiol Biochem 2015;35:957968.

24 Li J, Zhou F, Zheng T, Pan Z, Liang X, Huang J, Zheng L, Zheng Y: Ovarian Germline Stem Cells (OGSCs) and the Hippo Signaling Pathway Association with Physiological and Pathological Ovarian Aging in Mice. Cell Physiol Biochem 2015;36:1712-1724.

-25 Terraciano P, Garcez T, Ayres L, Durli I, Baggio M, Kuhl CP, Laurino C, Passos E, Paz AH, Cirne-Lima E: Cell therapy for chemically induced ovarian failure in mice. Stem Cells Int 2014;2014:720753.

26 Zhang H, Liu L, Li X, Busayavalasa K, Shen Y, Hovatta O, Gustafsson JA, Liu K: Life-long in vivo cell-lineage tracing shows that no oogenesis originates from putative germline stem cells in adult mice. Proc Natl Acad Sci U S A 2014;111:17983-17988.

27 Yuan J, Zhang D, Wang L, Liu M, Mao J, Yin Y, Ye X, Liu N, Han J, Gao Y, Cheng T, Keefe DL, Liu L: No evidence for neo-oogenesis may link to ovarian senescence in adult monkey. Stem Cells 2013;31:2538-2550.

28 Lu Z, Wu M, Zhang J, Xiong J, Cheng J, Shen W, Luo A, Fang L, Wang S: Improvement in Isolation and Identification of Mouse Oogonial Stem Cells. Stem Cells Int 2016;2016:2749461.

29 Gheorghisan-Galateanu AA, Hinescu ME, Enciu AM: Ovarian adult stem cells: hope or pitfall? J Ovarian Res 2014;7:71.

-30 Reizel Y, Itzkovitz S, Adar R, Elbaz J, Jinich A, Chapal-Ilani N, Maruvka YE, Nevo N, Marx Z, Horovitz I, Wasserstrom A, Mayo A, Shur I, Benayahu D, Skorecki K, Segal E, Dekel N, Shapiro E: Cell lineage analysis of the mammalian female germline. PLoS Genet 2012;8:e1002477.

-31 Dunlop CE, Telfer EE, Anderson RA: Ovarian germline stem cells. Stem Cell Res Ther 2014;5:98.

-32 Pan Z, Sun M, Li J, Zhou F, Liang X, Huang J, Zheng T, Zheng L, Zheng Y: The Expression of Markers Related to Ovarian Germline Stem Cells in the Mouse Ovarian Surface Epithelium and the Correlation with Notch Signaling Pathway. Cell Physiol Biochem 2015;37:2311-2322.

33 White YA, Woods DC, Takai Y, Ishihara O, Seki H, Tilly JL: Oocyte formation by mitotically active germ cells purified from ovaries of reproductive-age women. Nat Med 2012;18:413-421.

-34 Pacchiarotti J, Maki C, Ramos T, Marh J, Howerton K, Wong J, Pham J, Anorve S, Chow YC, Izadyar F: Differentiation potential of germ line stem cells derived from the postnatal mouse ovary. Differentiation 2010;79:159-170.

-35 Meng F, Chen C, Wan H, Zhou Q: [Advances of lentiviral vectors]. Zhongguo Fei Ai Za Zhi 2014;17:870-876.

-36 Morris KV, Rossi JJ: Lentivirus-mediated RNA interference therapy for human immunodeficiency virus type 1 infection. Hum Gene Ther 2006;17:479-486.

37 Xu CM, Wan FS: [Hippo signaling pathway in mammals: a new therapeutic target for tumors]. Yi Chuan 2012;34:269-280.

- 38 Zhang ZW, Men T, Feng RC, Li YC, Zhou D, Teng CB: miR-375 inhibits proliferation of mouse pancreatic progenitor cells by targeting YAP1. Cell Physiol Biochem 2013;32:1808-1817. 